A N N A L E S Annales de Bretagne et des Pays de l'Ouest

Anjou. Maine. Poitou-Charente. Touraine

113-4 | 2006

Varia

\title{
De la répulsion à la spéculation
}

La transformation du foncier littoral en Bretagne-Sud et en Vendée (1800-1939)

Johan Vincent

\section{OpenEdition}

1 Journals

Édition électronique

URL : http://journals.openedition.org/abpo/535

DOI : $10.4000 / a b p o .535$

ISBN : 978-2-7535-1504-8

ISSN : 2108-6443

Éditeur

Presses universitaires de Rennes

Édition imprimée

Date de publication : 30 décembre 2006

Pagination : $35-48$

ISBN : 978-2-7535-0405-9

ISSN : 0399-0826

\section{Référence électronique}

Johan Vincent, « De la répulsion à la spéculation », Annales de Bretagne et des Pays de l'Ouest [En ligne], 113-4 | 2006, mis en ligne le 30 décembre 2008, consulté le 01 mai 2019. URL : http:// journals.openedition.org/abpo/535; DOI : 10.4000/abpo.535 


\title{
De la répulsion à la spéculation La transformation du foncier littoral en Bretagne-Sud et en Vendée (1800-1939)
}

\author{
Johan VINCENT \\ Docteur en Histoire, \\ CERHIO - Université de Bretagne-Sud \\ GEOMER - Université de Bretagne-Occidentale
}

D'aucuns déplorent la volonté de leurs concitoyens de posséder un petit bout de littoral en propre, qui conduirait à une urbanisation massive, préjudiciable au littoral. Certains auteurs sont particulièrement critiques à l'égard de cet environnement " égaré ${ }^{1}$ ». Le bord de mer risque ainsi d'être bordé d'une ligne de maisons et d'immeubles si aucune volonté publique ne s'interpose. La Loi littorale, votée en 1986, est souvent citée comme un progrès dans le combat pour l'endiguement des constructions littorales. En se transformant, le rivage maritime perdrait, en effet, tout son intérêt. Cet intérêt est pourtant relativement récent : il remonte au XVIII ${ }^{\mathrm{e}}$ siècle, quand les notables se sont entichés de la mer. Désireux de se confronter à la nature, ils se sont promenés au bord de l'eau. Servi par un nouveau discours médical qui faisait du séjour à la mer un remède efficace, le bord de mer est devenu attractif. Auparavant, ce n'était qu'un bout du monde, à l'écart des zones occupées par l'homme. S'il faisait partie des espaces utilisés par la communauté, il demeurait un site marginal. En deux siècles, la situation s'est totalement inversée. Comment est-on passé de la répulsion à la spéculation sur ce même espace?

L'aspect foncier du littoral est un sujet relativement peu étudié, mais les problèmes d'aujourd'hui motivent la recherche en sciences sociales. L'aspect foncier s'intègre dans la notion de territoire, particulièrement étudiée par la géographie. Une majorité des travaux portent sur les conséquences de l'urbanisation qui aboutit à figer le territoire. Le littoral reste un espace aux frontières variables : le trait de côte est fluctuant, selon les régions et les époques, la mer pouvant créer ou envahir des terrains. Face à ces questions, la géographie a des réponses à apporter, mais elle réflé-

1. LESCOAT, Jacques, La Bretagne ou l'environnement égaré; Essai sur l'organisation de l'espace en France à partir du cas breton, Spézet, Éditions Nature et Bretagne, 1996, 164 p. 
chit peu sur la genèse de l'urbanisation et sa motivation, ou alors de manière téléologique - l'urbanisation apparaît naturelle et rien n'aurait pu l'arrêter - ainsi dans l'ouvrage de Sylvie Caillé, Les Côtes sableuses du $X I X^{e}$ siècle à nos jours ${ }^{2}$. De leur côté, les historiens ont eu tendance à délaisser l'étude foncière du littoral : Nadine Vivier dans son ouvrage sur les biens communaux ne traite pas le littoral comme un espace à part entière ${ }^{3}$. Il faut avouer que la diversité des littoraux ne facilite pas la tâche. Si l'aspect social du nouvel attrait du bord de mer est étudié, notamment depuis l'ouvrage d'Alain Corbin sur Le Territoire du vide ${ }^{4}$, il apparaît que l'aspect foncier de ce territoire a, pour l'instant, suscité peu de synthèses (tel le travail de Gaëlle Delignon sur Saint-Malo et Paramé ${ }^{5}$ ) permettant de comprendre ce passage à la spéculation.

Différentes dynamiques ont agi pour permettre cette révolution. Le bord de mer présentait autrefois un aspect différent de celui que nous connaissons ou que nous imaginons. Comprendre comment il se présentait permet de saisir quel pouvait être l'usage d'une telle étendue avant qu'elle ne soit investie par l'activité balnéaire. Étudier un territoire implique d'analyser l'usage qui en est fait. Si aujourd'hui, le littoral ne peut ressembler qu'à un espace où l'on habite, où l'on se divertit et où l'on se promène, c'est que l'homme a œuvré en ce sens. Sur le littoral, une vigne ou tout autre espace agricole relève aujourd'hui de la curiosité. Les populations littorales ont donc leur importance dans ce processus : la demande seule ne suffit pas à créer l'offre. Le contexte a cependant une influence considérable. Les évolutions sont multiples au cours du XIXe siècle. L'État tâche de maîtriser le territoire avec des politiques de grands travaux (boisements) et la mise en place du cadastre pour l'impôt; le poids de la communauté villageoise se dilue avec la fin de l'usage des communaux car nul n'est plus tenu à l'indivision. Le bord de mer est une conquête qu'il convient de matérialiser.

\section{Le littoral, paysage désertique}

Au début du XIXe siècle, les paysages de bord de mer sont bien différents de ceux d'aujourd'hui. Ils rappellent le désert, où l'installation humaine est généralement précaire, mais où l'homme n'est toutefois pas complètement absent.

2. CAILlÉ, Sylvie, Les Côtes sableuses du XIXe siècle à nos jours (Loire-Atlantique et Vendée), Nantes/Laval, Siloë, 2003, 143 p.; si la partie sur l'implantation des stations balnéaires est discutable, le reste de l'ouvrage n'en demeure pas moins de bonne facture.

3. VIVIER, Nadine, Propriété collective et identité communale; les biens communaux en France 1750-1914, Paris, Publications de la Sorbonne, 1998, 352 p.

4. CORBIN, Alain, Le Territoire du vide; l'Occident et le désir de rivage (1750-1840), Paris, Aubier, coll. " Historique ", 1988, 399 p.

5. DeLIGNON, Gaëlle, Saint-Malo-Paramé; Urbanisme et architecture balnéaire (1840-1940), Rennes, PUR, 1999, 252 p. 


\section{Loin de la présence humaine}

Le bord de mer au XIX ${ }^{\mathrm{e}}$ siècle est un lieu où l'homme apparaît comme un étranger. En 1823, du côté d'Escoublac, à l'emplacement où se construira La Baule, " [...] on arrive au pied de ces montagnes, dont le sommet, aperçu de si loin, se découvre dans toute sa nudité. On gravit avec peine ce sol qui fuit sous les pieds et qui n'offre aucune végétation qui aide à escalader sa pente verticale. On arrive sur le point le plus élevé, et d'autres sommets aussi stériles et séparés par d'autres vallées se prolongent jusqu'à la mer qui vient, en bouillonnant, recouvrir la rive de dépôts plus récents. De là, jusqu'au Pouliguen, à une lieue à l'ouest; au nouveau bourg d'Escoublac, à un quart de lieue au nord, et au village de Pornichet, à une demie lieue à l'est, la vue ne trouve partout que le même aspect : ce sont les sables et la mer, vous êtes là seul entre une plage déserte et des dunes abandonnées ${ }^{6}$ ". Il faut bien du romantisme pour apprécier ces espaces nus. Pour Hippolyte Etiennez, en 1858, " l'aspect de ces dunes éveille dans l'âme les impressions les plus tristes; c'est, en petit, l'image la plus complète du désert, avec son silence, sa solitude et sa stérilité. Continuellement modifiée par l'action du vent, cette arène mobile, qui a aussi elle ses tempêtes, affecte parfois les formes les plus singulières. Tantôt ce sont les crêtes escarpées comme des brèches dont le temps aurait arrondi les contours, tantôt des murs verticaux dont le passage d'un oiseau suffit pour déterminer la chute $[\ldots]^{7}$ ".

Toutes les zones dunaires, avant d'être fixées, sont à peu près dénuées de présence humaine permanente. Des marais et des étangs stagnent souvent à l'arrière du cordon dunaire. Ils renforcent le peu d'attrait de cet environnement difficile. Encore en 1890, « la plage de Carnac était absolument déserte. Une ligne de dunes la bordait le long du continent. Les eaux avaient formé, en arrière, des champs fangeux et des marais qu'on avait plus ou moins aménagés pour la production de sel. Une bande tourbeuse séparait ainsi la plage du coteau à pente douce qui monte jusqu'au pied du tumulus Saint-Michel et à quelques centaines de mètres plus à l'ouest jusqu'au bourg commerçant de Carnac $[\ldots]^{8}$ ". Le conseil général de Charente-Inférieure en 1863 plaint les pauvres naufragés qui « [...] se trouvent jetés sur une plage déserte, privés de secours, mourant de faim, de froid et de fatigue, et éloignés de plusieurs kilomètres de toute habitation ${ }^{9}$ ". Quelques villages existent à proximité du bord de mer, mais ils sont rares et généralement disposés à proximité d'une côte rocheuse qui les protège des méfaits du vent et

6. RICHER, Édouard, Voyage pittoresque dans le département de la Loire-Inférieure, Paris, Éditions du Palais royal, 1974 [1 1 re éd. 1823], p. 553-554.

7. ETIENNEZ, Hippolyte, Guide du voyageur à Nantes et aux environs, Nantes, Éditions Petitpas, $1858,328 \mathrm{p}$.

8. GALLOuÉDEC, L., "Au pays des mégalithes : le bourg de Carnac et sa plage (Morbihan) ", La Revue du Touring Club de France, 1932, p. 208.

9 . Compte-rendu du Conseil général de 1863, cité par Patricia Toucas, « La gestion de l'espace littoral par le département de Charente-Inférieure sous le Second Empire (18521870) ", dans MalEzIEUX, J. (dir.), Le Milieu littoral : actes du $124^{e}$ congrès national des sociétés historiques et scientifiques (Nantes 1999), Paris, CTHS, 2002, p. 300. 
du sable. Le village de Trez Bian, à Bénodet, se situe à proximité de la plage du Trez, mais il demeure le plus à l'est de cette plage, protégé par la pointe Saint-Gilles. Les habitations les plus courantes que l'on peut alors trouver sur le littoral atlantique sont les cabanes de douane, bien souvent abandonnées depuis la fin du blocus de la période napoléonienne.

\section{Les déserts de sable}

Si l'installation permanente de l'homme y est généralement impossible, il n'a cependant pas complètement abandonné le bord de mer. L'espace des dunes est traversé par une multitude de sentiers permettant d'atteindre la mer et ses ressources. Malgré l'absence d'un port en de nombreux endroits, quelques habitants possèdent parfois, pour pêcher, un bateau, tiré sur la plage. D'autres entretiennent des pêcheries, constructions qui emprisonnent le poisson à marée basse, les plus miséreux cueillent les coquillages que la mer apporte. Il leur faut donc bien traverser la zone dunaire. La mer est utile également aux populations de l'intérieur. À Saint-Jean-de-Monts, un chemin qui va à la mer est ainsi classé comme chemin vicinal en 1856 par arrêté préfectoral afin de pourvoir aux intérêts de la commune. Il demeure en l'état de chemin sablonneux et mouvant jusqu'en 1867, date où est décidée la construction d'une route. Le conseil municipal indique qu'il est essentiel aux activités traditionnelles, " pour pouvoir [accéder] en tout temps à la côte avec chevaux et voitures; soit pour le transport des matériaux qui sont chaque année employés pour l'entretien des routes vicinales de la contrée, soit pour le transport du varech [ou goémon] qui sert d'engrais aux terres du littoral, soit pour le transport des épaves qui échouent à la côte $[\ldots]^{10}$ ". Quand la terre le permet, le littoral sert aussi au pâturage. Jane Auffret-Quintin se souvient à Crozon qu'elle gardait les vaches avec d'autres enfants; le décor qu'elle préférait était le grand champ près de la mer face au large, mais elle précise qu'on s'y donnait rendez-vous par temps calme, quand le vent de mer oubliait de souffler ${ }^{11}$. Certaines vaches s'installent sur les dunes, où une maigre végétation s'épanouit, comme à Damgan où elles sont photographiées au début du Xxe siècle.

Les dunes sont cependant plus ou moins protégées, car elles sont le premier obstacle face à la mer. Madame Lebleis, propriétaire des marais de Brémoguer à Loctudy, s'inquiète de la dégradation de la dune qui est devenue assez importante pour compromettre l'existence de sa propriété. Ces dégradations sont dues à l'enlèvement du sable des dunes par les cultivateurs (qui se procurent ainsi un amendement marin), au dépôt de goémon et au passage des charrettes sur les chemins pratiqués à travers les dunes. L'ingénieur ordinaire des Ponts-et-Chaussées, M. Juncke, rappelle qu'il est nécessaire de bien entretenir les chemins et d'obliger ceux qui ont

10. Délibération municipale de Saint-Jean-de-Monts, du 27 septembre 1867.

11. AufFret-Quintin, Jane, Mon beau pays perdu, 1989, p. 31 ; l'auteur a 8 ans, en 1914, année où se déroulent ces faits. 
déposé du goémon à couvrir les lieux de dépôt de pierres ou de fascines (assemblage de branchages) de manière à éviter toute dégradation et à favoriser l'élévation du niveau de la dune ${ }^{12}$. Il réitère ces recommandations pour l'ensemble du littoral de Loctudy, où les charrettes des cultivateurs sillonnent la dune dans tous les sens, sans se rendre véritablement compte des déprédations que leurs passages occasionnent, et notamment des risques d'envahissement d'un ancien marais situé à l'arrière de la dune ${ }^{13}$. Celle-ci peut aussi accueillir des exploitations agricoles. La vigne est régulièrement plantée en Vendée et Loire-Atlantique sur le bord de mer. Elle est susceptible de se faire envahir et, en 1897 à Saint-Brévin M. Martel perd en dix ans plus d'un hectare de vigne et de bois de pins. Sa vigne sert également de lieu où se changent les baigneurs, à son grand dam, ce qui montre la proximité de l'exploitation par rapport à la mer ${ }^{14}$. L'orge, les fèves, les aulx, les oignons, l'asperge et les pommes de terre sont aussi des cultures possibles, si le sable et le sel, propulsés par le vent, les épargnent. Les dunes fixées permettent l'implantation de constructions industrielles : elles forment des points hauts nécessaires aux moulins à vent. Ces déserts de sable ne sont donc pas totalement vides.

\section{Les nouveaux intérêts d'un territoire}

Au cours du XIX ${ }^{\mathrm{e}}$ siècle, le littoral est transformé, sous l'action simultanée des touristes et de l'État. Ces modifications vont renforcer la particularité de ce territoire.

\section{La fixation des terres}

La fixation des terrains littoraux est décidée sous l'Empire. Les villages littoraux avaient connu bien des vicissitudes avec les tempêtes de sable : les bourgs d'Escoublac et de Saint-Brévin sont les exemples les plus cités d'un nécessaire repli de l'homme face à l'avancée des dunes. Napoléon Ier décide par décret du 14 décembre 1810 la fixation des dunes par ensemencement. La mesure est particulièrement volontaire : dans le cas d'une propriété privée, l'article 5 prévoit que "si lesdits particuliers ou communes se trouvaient hors d'état d'exécuter les travaux commandés, ou s'y refusaient, l'administration publique pourra être autorisée à pourvoir à la plantation des dunes à ses frais; alors elle conservera la jouissance des dunes [...] jusqu'à l'entier recouvrement des dépenses qu'elle aura été dans le cas de faire, et des intérêts; après quoi lesdites dunes retourneront aux

12. Arch. dép. du Finistère, 4 S 137, Rapport de M. Juncke, ingénieur ordinaire des Pontset-Chaussées, sur une réglementation des extractions, du 30 octobre 1871.

13. Arch. dép. du Finistère, 4 S 137, Rapport de M. Juncke, ingénieur ordinaire des Pontset-Chaussées, sur la réglementation de l'enlèvement des sables et le dépôt de goémons, du 10 janvier 1872 .

14. Arch. dép. de Loire-Atlantique, 536 S 22, Lettre de M. Martel (Saint-Brevin) au préfet de Loire-Inférieure, du 12 septembre 1897. 
propriétaires, à charge d'entretenir convenablement les plantations ». Les résistances, dans un premier temps, sont toutefois rares et la plupart des utilisateurs des dunes préfèrent se retirer - officiellement mais pas officieusement - sur des terres plus en arrière. À La Baule, " du côté des particuliers, seule une dame Larragon, veuve Legruger, propriétaire d'une ferme en partie envahie (métairie de la Jau [en arrière de l'actuel La Bauleles-Pins]) manifesta une opposition sérieuse et résolut de conserver ses terres pour en retirer elle-même en l'état les produits qu'elle pourrait. On prit alors les mesures nécessaires [...] et satisfaction fut donnée à l'opposante, qui, d'ailleurs, se vit bientôt contrainte de tout abandonner à la suite d'un envahissement définitif [en 1815] ${ }^{15}$ ".

L'échec des plantations est cependant courant et il faut de nombreuses années avant que les dunes ne soient fixées. À Escoublac, le comte de Sesmaisons obtient en 1818 la concession des 642 hectares de dunes pour trente ans, mais il ne parvient pas à lutter contre le sable et le vent. Il se heurte également à l'hostilité des habitants à qui l'État a retiré la jouissance d'une partie de la commune, en y interdisant le pacage des animaux. Il vend sa concession en 1834 et 1836 à divers entrepreneurs, qui ne réussissent pas plus, à partir des années 1840, à y implanter des champs de luzerne. Le décret de 1810 ne se met donc en application qu'au cours du XIX ${ }^{\mathrm{e}}$ siècle. En 1864 et à nouveau en 1869, le conseil municipal de Saint-Gilles-sur-Vie récrimine contre $\mathrm{M}$. Bascher, propriétaire de la garenne de Retz (flèche dunaire qui protège le port), car depuis 1845 et sa première demande pour planter de la vigne et des arbres résineux - tentative qui a échoué -, le sable continue de se déposer dans le chenal ${ }^{16}$. L'administration tarde elle aussi parfois à lancer cette politique de grands travaux. Les dunes du pays de Monts ne sont couvertes qu'à partir des années 1850. La partie située entre le sémaphore et Kerlosken à Beg-Meil n'est fixée qu'en 1907, quand « des hommes soucieux du bien public tels que Louis Hemon et Monsieur Bénac entreprirent de faire planter la dune [... avec pour] but [...] d'en fixer le sol et surtout de procurer aux promeneurs de l'ombrage et un lieu de repos ". Malgré toute l'expérience accumulée au XIX ${ }^{\mathrm{e}}$ siècle, ces plantations ont dû être recommencées plusieurs fois ${ }^{17}$.

\section{La parcellisation des terres}

La fixation des dunes s'accompagne d'une cadastration, décidée par la loi du 15 septembre 1807 mais essentiellement mise en œuvre sous la Monarchie de Juillet. Cette cadastration a probablement encouragé les plantations, car il n'était pas question de dépenser de l'argent pour le terrain d'un autre. Elle bouleverse pourtant, elle aussi, les habitudes des

15. Arch. dép. de Loire-Atlantique $532 \mathrm{~S}$ 11, Revendications des dunes d'Escoublac; Mémoire en faveur de l'État français, Nantes, Imprimerie A. Dugas et Cie, 1904, p. 5-6.

16. Délibérations municipales de Saint-Gilles-sur-Vie, des 9 mai 1864 et 26 décembre 1869.

17. Délibération municipale de Fouesnant, du 21 août 1927. 
autochtones : l'espace côtier sans contrainte laisse la place à des zones délimitées. Il n'est plus question d'aller empiéter, notamment avec les bestiaux, sur les propriétés maintenant privées. Pierre-Yannick Legal explique d'ailleurs, dans un article sur la plantation de la forêt littorale d'Olonne, que « ce sont moins les éléments naturels qu'il fallut combattre, que les résistances des propriétaires et des usagers, en une période où le droit de propriété était mal assis ${ }^{18}$ ". Suite à l'obligation faite aux propriétaires de devoir fixer leurs terrains, l'État possède une superficie importante du littoral, qu'il délimite, parfois avec peine, qu'il concède ou dont il s'occupe. Certaines concessions sont transformées en propriétés privées, comme à La Baule où Yves Berthault devient propriétaire d'une concession de près de six cents hectares de dunes sur décision du président de la République en $1884^{19}$, mais le cas semble rare. Les autorités tâchent de préserver quelques espaces communs. Des chemins sont dessinés et des parcs à goémon sont sauvegardés, afin qu'un accès et un usage de la mer soient toujours possibles. Lors de l'aliénation des terrains communaux de SaintBrévin, l'expert désigné par le tribunal, M. Padioleau, procède " de concert avec la municipalité [...] à l'établissement des chemins indispensables aux habitants pour aborder le rivage de la mer et arrête qu'il serait laissé à leur extrémité, sur le chemin de ceinture, des vagues ou places pour disposer les engrais (goémons); [il réserve] tout le long du rivage un chemin de ceinture d'au moins 12 mètres de largeur, à partir des plus hautes mers ${ }^{20}$ ".

Ces délimitations n'ont cependant qu'une valeur symbolique pendant une partie du XIX ${ }^{\mathrm{e}}$ siècle. En effet, certains autochtones n'hésitent pas à s'implanter sur des terrains qui ne leur appartiennent pas, notamment sur les terrains domaniaux. Le défaut de surveillance permet l'appropriation illégitime. À Saint-Jean-de-Monts, " depuis les opérations cadastrales, des particuliers se sont emparés sur la lisière des dunes de terrains d'une qualité relativement supérieure, les ont entourés de talus en terre et les ont ensuite mis en culture. D'autres n'ont pas craint [...] d'enclore au milieu même de la propriété domaniale les terrains qui leur convenaient le mieux et d'y établir des chaumières dites Bourines pour leur servir de logement $^{21}$ ". Pour le curé, en 1918, il ne fait d'ailleurs guère de doute qu'avant la plantation de la forêt, à la fin des années 1850, n'importe qui pouvait s'installer dans la dune : l'État s'empara « de tous les terrains qui étaient res nullius et respecta les droits de ceux qui s'étaient taillé des enclaves, sous la double condition de faire cadastrer leur terrain et de payer le

18. LEGAL Pierre-Yannick, «La plantation de la forêt d'Olonne; Droit de propriété privée et contraintes d'utilité publique dans la première moitié du XIX ${ }^{\mathrm{e}}$ siècle ", Recherches vendéennes $\mathrm{n}^{\circ} 12,2005$, p. 313

19. ChARLES, Alain, "Dans le doux dédale des dunes ", 303, Arts, recherches et créations, $1^{\mathrm{er}}$ trim. 1994, p. 32.

20. Arch. dép. de Loire-Atlantique, 532 S 32, Lettre du directeur de l'Enregistrement, des Domaines et du Timbre, au préfet de Loire-Inférieure, du 4 novembre 1896.

21. Arch. dép. de Vendée, 7 M 199, Procès-verbal de reconnaissance du sous-inspecteur des Forêts sur la forêt de Saint-Jean-de-Monts, du 20 décembre 1866. 
modique impôt de un franc par hectare ${ }^{22}$ ». Sans réelle autorité pour faire respecter les limites de propriété, le cadastre (utilisé pour l'impôt) demeure des plans de papier. Tant que les terrains n'ont pas d'usage propre, le flou perdure : la limite entre Saint-Hilaire-de-Riez et Saint-Jean-de-Monts ne devient une préoccupation que quand des constructions s'élèvent et qu'on ne sait sur quelle commune elles sont exactement bâties. Le bornage, réclamé en 1928, n'est toujours pas terminé en $1930^{23}$.

\section{L'achat des terres}

L'intérêt pour le littoral, apparu au xvIII siècle, conduit les urbains aisés à chercher à se confronter à la nature. En même temps, le discours médical met en avant le séjour au bord de mer qui permet de rompre avec un environnement vicié. Ceci entraîne des séjours réguliers, d'abord sur les côtes normandes (plus proches de Paris) et provençales (sur le trajet de l'Italie, étape avant de franchir les Alpes), puis sur les autres côtes françaises à partir du début du xix ${ }^{\mathrm{e}}$ siècle. Si les premiers séjours ont été l'occasion de demeurer chez l'habitant ou à l'hôtel, une partie des baigneurs décident ensuite de faire construire. Ils s'implantent au plus près de la mer, secteur généralement libre d'installation. Ces constructions sont d'ailleurs ressenties comme bénéfiques : «Des établissements de bains se sont élevés sur presque toutes les plages; des sables arides se sont convertis en verdoyants chalets, et nos rudes et intéressantes populations maritimes ont recueilli, les premières, les bénéfices de ces heureuses transformations ${ }^{24}$. " Les propriétaires locaux comprennent leur intérêt. Le maire de La Tranche-sur-Mer déclare, en 1904, vouloir vendre des terrains communaux proches de la mer pour stopper l'envahissement du sable dû au vent et pour permettre à des étrangers d'acquérir " pour faire construire [car] si on refuse de les leur céder aujourd'hui, ils iront faire construire sur une autre plage ${ }^{25}$ \%. Le conseil municipal de Noirmoutier participe à cette concurrence entre les stations en réclamant la mise à disposition des terrains situés au bord de la mer sur lesquels pourraient être édifiées de nouvelles villas ${ }^{26}$.

Les terrains littoraux ne sont pas toujours disponibles et les baigneurs ont su parfois profiter d'autres opportunités. L'élan de construction, qui commence à partir de la seconde moitié du XIXe siècle, coïncide en Bretagne avec la vente des communaux (quasiment inexistants en Vendée). Si la vente des dunes de Saint-Brévin prescrite par le ministre des Finances en

22. Bulletin paroissial de Saint-Jean-de-Monts, de juillet 1918.

23. Délibérations municipales de Saint-Jean-de-Monts, des 10 juin 1928 et $1^{\mathrm{er}}$ juin 1930.

24. Service historique de la Marine de Vincennes, $\mathrm{CC}^{5} 217$, « Des bains de mer et de la propriété des rivages de la mer ", Journal officiel de l'Empire français, du 8 avril 1870.

25. Arch. dép. de Vendée, 1 O 799, Rapport du maire de La Tranche sur l'aliénation de terrains communaux, du 20 décembre 1904.

26. Délibération municipale de Noirmoutier, du 8 octobre 1907; a contrario, il demande le 30 septembre 1908 que les Domaines ne vendent plus au Bois de la Chaise (repris le 19 août 1911). 
1841, s'éternise devant les tribunaux, jusqu'à l'adjudication aux enchères, en 1859 et 1860, entre les différents intéressés ${ }^{27}$, les communs de Préfailles sont promptement partagés en 1845 , par tirage au sort ${ }^{28}$. Ce mouvement est encouragé par la loi du 6 décembre 1850, qui simplifie la procédure pour le partage des terres vaines et vagues de Bretagne. Comme le remarque Nadine Vivier dans son ouvrage sur les communaux en France, l'essentiel des partages dans cette région ont lieu entre 1850 et $1880^{29}$. Les terres vaines et vagues de Tréboul sont divisées entre les propriétaires à la suite du jugement du Tribunal civil de Quimper du 18 mars $1872^{30}$. Cinq propriétaires au Poullan (dont dépend à l'époque Tréboul), notamment Gustave Le Guillou Penanros, négociant à Douarnenez, parviennent à faire partager les terres vaines et vagues; la famille Le Guillou Penanros saura faire fructifier au cours de la première moitié du xx siècle les terrains qu'elle a reçus. Le manque d'argent nécessite souvent la vente de terrains. Lorsque se constitue la Société civile des Bains de mer et terrains de Saint-Brévin en 1883, aucune des propriétés achetées à partir de 1881 n'appartient à des Brévinois, ce qui implique des ventes au cours des vingt années précédentes ${ }^{31}$. Si le maire de La Tranche-sur-Mer désire vendre des terrains communaux en 1904, c'est pour avoir les moyens de construire le logement de l'instituteur. L'État vend également : la situation critique du budget, en novembre 1926, le conduit à aliéner les huit hectares de dunes qu'il possède à Beg-Meil, au grand dam de la municipalité et du conseil général du Finistère ${ }^{32}$. Le conseil municipal de Notre-Dame-de-Monts profite d'une situation similaire en 1923 pour acheter et lotir une portion de la forêt domaniale qu'elle espérait exploiter pour les bains depuis plus de vingt ans ${ }^{33}$.

\section{La course vers le front de mer}

La mise en vente des terrains du bord de mer permet aux touristes de s'implanter dans les espaces qu'ils recherchent. L'activité balnéaire modifie la valeur des terrains. Le bord de mer devient le lieu où il faut s'implanter.

27. Arch. dép. de Loire-Atlantique, 532 S 32, Lettre du Directeur de l'Enregistrement, des Domaines et du Timbre, au Préfet de Loire-Inférieure, du 4 novembre 1896.

28. Arch. dép. de Loire-Atlantique, 2 O 136/6, Lettre du Directeur de l'Enregistrement, des Domaines et du Timbre, au Préfet de Loire-Inférieure, du 24 décembre 1932; Louis Emery retrace cette histoire dans Les Communs de Préfailles, Paimbœuf : Imprimerie Coyaud, 1935, 26 p.

29. VIVIER Nadine, op. cit., p. 254-256.

30. Arch. dép. du Finistère, 15 U 14/84, Jugement civil du tribunal de Quimper, du 18 mars 1872.

31. Arch. dép. de Loire-Atlantique, 4E 51/97, Dépôt des statuts de la Société civile des Bains de mer et terrains de Saint-Brévin, du 3 mars 1883; la superficie totale achetée dépasse les 200 hectares et la majorité des précédents propriétaires sont nantais.

32. Arch. dép. du Finistère, 4 S 29, Rapport de M. Fatou, inspecteur principal des Eaux et Forêts, sur le vœu du conseil municipal de Fouesnant tendant à l'abandon du projet d'aliénation des dunes, du 31 août 1927.

33. Délibérations municipales de Notre-Dame-de-Monts, des 7 décembre 1902 et 19 août 1923. 


\section{Voir la mer}

La proximité de la mer a toujours eu une importance considérable. En 1864, un particulier, M. Crusson, demande la concession de terrains domaniaux à La Turballe. Ce village, port de pêche en développement, ne connaît l'attraction balnéaire que grâce à la proximité du Croisic, première station balnéaire historique de la presqu'île de Guérande. Or, l'ingénieur des Pontset-Chaussées apprend que ce projet consiste en fait à tracer une route entre les propriétés existantes et la mer et à construire une série de maisons de l'autre côté du chemin : pour ce fonctionnaire, le projet «[...] ne pourrait se réaliser qu'en masquant les constructions actuelles, et en les privant du voisinage immédiat de la plage qui constitue une grande partie de leur valeur ${ }^{34}$ ". Pour une maison construite dans une station balnéaire, cette qualité d'avoir la vue sur la mer peut donc être essentielle, d'autant que certains en profitent pour les proposer à la location. Quand la mairie des Sables-d'Olonne décide d'aliéner une place sur le bord de la mer en 1878, Théophile Leblanc, comme d'autres propriétaires voisins, s'offusque, car " [...] la maison qu'il possède se trouvant au-dessus et en face de la place Navarin, si celle-ci étant vendue par la ville pour y faire des constructions, sa maison se trouverait très gênée et perdrait beaucoup de sa valeur, n'ayant plus comme aujourd'hui la vue sur mer; et que l'amoindrissement inévitable du revenu annuel qu'il en retire serait pour lui un préjudice considérable ${ }^{35}$ ». Plus que sa situation sur le bord de mer, c'est la vue sur mer qui semble importer. Certains propriétaires ont d'ailleurs fait les comptes : selon M. Morvan de Plobannalec, propriétaire d'une parcelle de 5 à 6 ares à Loctudy, la vue sur les baies de l'llle-Tudy et de Bénodet entraîne une plus-value de plus de deux mille francs (multiplication par 5 à 6 de la valeur du terrain) ${ }^{36}$.

La vue sur mer réclame des protections particulières. Le conseil municipal du Pouliguen délibère en 1927 « contre l'édification sur les terrains de la plage du Nau de toute construction susceptible de gêner la vue qu'ils ont actuellement sur la mer ${ }^{37}$ ". Ce problème s'inscrit dans le débat sur l'urbanisation (abusive ou avilissante) du trait de côte, débat qui émerge, en certains endroits, dès les années 1870. Le conseil municipal de Fouesnant récrimine en 1927 contre l'État qui désire vendre huit hectares trente centiares dont il est propriétaire : les acheteurs vont bâtir des maisons et des murs de clôture et " les touristes qui venaient nombreux pour admirer cette région pittoresque abandonneront la plage de Beg-Meil parce qu'ils ne pourront plus jouir de la vue sur mer qui sera bouchée par les constructions et

34. Arch. dép. de Loire-Atlantique, 532 S 39, Rapport de l'ingénieur ordinaire des Pontset-Chaussées sur la concession demandée par le Sr Crusson, du 14 avril 1864.

35. Arch. dép. de Vendée, 1 O 612, Enquête de commodo et incommodo relative aux projets d'acquisition d'une maison située au bas de la rue Navarin et d'aliénation de la place portant ce nom, Les Sables-d'Olonne, 20 août 1878.

36. Arch. dép. du Finistère, 4 S 65, Lettre de M. P. Morvan au préfet du Finistère, du 12 mars 1903; ses parents auraient en effet trouvé des acheteurs à 3000 francs, tandis qu'en tant que terre enclavée, sa propriété ne vaudrait plus que 100 francs l'are.

37. Délibération municipale du Pouliguen, du 3 juin 1927. 
les murs ${ }^{38}$ ". Remarquons toutefois que la commune n'a rien à gagner dans la vente, ce qui lui permet d'avouer franchement son aversion pour ce projet. Il est également possible d'interdire toute construction future entre la villa et la mer, en introduisant une servitude de vue dans une clause de vente. À Saint-Malo, le banquier Hébert, principal lotisseur de la station balnéaire, avait eu cette prudence pour sa villa. Une servitude de vue intervient à Douarnenez, dans le quartier du Petit-Port, quand Charles Chancerelle, en 1904, demande de clore ses propriétés en achetant un terrain communal. Le conseil municipal prend garde de prévenir l'acheteur que ce terrain est grevé en grande partie d'une servitude de vue, au profit de la propriété Queïnnec ${ }^{39}$. Les exemples en sont néanmoins rares, ce procédé semble peu utilisé car il faut être généralement propriétaire de la parcelle à vendre. Toujours se rapprocher de la mer n'est pourtant pas sans danger, car le trait de côte fluctue : en cinq ans, entre 1906 et 1911, certaines propriétés sont amputées de près de dix mètres à Saint-Brévin ${ }^{40}$. Pourtant, ce risque est largement sous-estimé en 1908, quand d'autres empiètent sur la propriété domaniale des lais de mer, obligeant l'administration " [...] au bornage des lais de mer [du Pointeau et de la Guerche], dont la valeur peut devenir très importante en raison du développement des stations balnéaires ${ }^{41}$ ". La mise en place du remblai permet de construire une ligne entre plage et terres, chassant l'idée de cette fluctuation du trait de côte tout en consolidant la valeur des propriétés qu'il longe, définitivement situées au plus près de la mer.

\section{Définir les limites de la plage}

Les limites de la plage méritent d'être définies particulièrement dans le cas des côtes sableuses. Ce problème ne se pose pas dans le cas des côtes rocheuses : la limite entre la plage et les terres est visible, les constructions s'élèvent en hauteur par rapport à la mer. Ce n'est pas le cas pour les côtes sableuses où plage et dunes s'alimentent mutuellement. Afin de limiter les nuisances résultant de l'apport de sable, les habitants projettent de construire des remblais, utiles à la promenade. Non seulement le remblai permet de délimiter la plage des terrains constructibles, mais il permet aussi de voir la plage. Le conseil municipal des Sables-d'Olonne s'enthousiasme dès 1830 : "Quel beau spectacle offrira notre magnifique plage où ses limites, si avantageusement tracées par la nature, seront fixées par des murs [devant le quartier des Corderies] ${ }^{42}$. " Le quartier des Corderies est

38. Délibération municipale de Fouesnant, du 21 août 1927.

39. Délibération municipale de Douarnenez, du 19 novembre 1904.

40. Arch. dép. de Loire-Atlantique, 536 S 22, Rapport de M. Dumas, conducteur des Ponts-et-Chaussées, sur la délimitation du rivage de la mer à Saint-Brévin, du 24 mai 1911.

41. Arch. dép. de Loire-Atlantique, 536 S 22, Avis de M. Maynard, ingénieur ordinaire des Ponts-et-Chaussées, sur le bornage des lais de mer sur le littoral de Saint-Brévin, du 13 février 1908.

42. Délibération du Conseil municipal des Sables-d'Olonne, du 8 mai 1830. 
en pleine urbanisation à cette époque. Le remblai construit, il ne reste plus à la station balnéaire qu'à l'animer. En 1905, "le soir, à l'époque des bains surtout, le Remblai s'illumine, sur un espace de trois mille mètres, de girandoles étincelantes et de rampes de feu qui miroitent dans les grandes ondes voisines et qui, avec des milliers de lumières embrasant fenêtres et balcons, donnent, dans cette vaste baie, l'illusion ravissante d'un immense feu d'artifice, fixé sur place par une baguette magique. Sous les rayons du soleil et aux premières heures de la nuit, tout le Remblai déploie une mise en scène véritablement prestigieuse et sans rivale en Europe ${ }^{43}$ ".

Malgré l'avantage évident de créer une promenade au bord de la mer, les remblais sont cependant relativement rares en Bretagne-Sud et Vendée avant l'Entre-deux-guerres. Le défaut d'ombre - particulièrement recherchée avant la mode du bronzage, dans les années 1920 -, la difficulté à lutter contre l'érosion littorale et l'existence de propriétés privées jusqu'aux limites des plus hautes mers dans certaines communes ont pu ralentir ce processus de fixation des limites de la plage. La nécessité de gagner rapidement sa villa en automobile a peut-être encouragé la multiplication des remblais; auparavant, automobilistes, cyclistes et piétons utilisaient la plage pour se rendre dans leurs propriétés sur le bord de mer, ce qui posait des problèmes de sécurité. De nombreux arrêtés municipaux, à partir des années 1900, limitent la vitesse des bicyclettes ou obligent les cyclistes à conduire leur moyen de locomotion à la main sur la plage. Le remblai de La Baule, terminé en 1930, et celui de Quiberon, sont ainsi praticables par les automobiles. M. Bonnisseau, ingénieur en chef des Ponts-et-Chaussées, milite en faveur du remblai de Pornichet construit dans le prolongement de celui de La Baule : " La plage de Pornichet s'amaigrit en effet assez sensiblement et si aucun obstacle n'est opposé à la progression de la mer, il est à craindre que les villas riveraines ne soient menacées et que la plage ne voit alors diminuer sa fréquentation et, partant, sa valeur. Celle-ci sera, au contraire, inévitablement augmentée par la construction du boulevard qui protégera la plage et en permettra l'exploitation dans de biens meilleures conditions. D'autre part, le manque d'une zone de circulation facile en bordure de plage de Pornichet [...] se fait de plus en plus sentir ${ }^{44}$."

Même les stations les plus modestes se dotent d'une promenade en bord de mer. Le remblai reste parfois une réalité plus théorique que réelle, espace en bordure de plage sur lequel a été décidée l'impossibilité de bâtir. À Fromentine, M. Marsat, inspecteur des Eaux et Forêts, rappelle que la dune littorale est restée propriété domaniale au moment de l'aliénation de 1907 : "Maintenant que ces terrains [en arrière] sont construits, cette bande littorale joue le rôle du "Remblai" aux Sables-d'Olonne, le public y

43. Rellno, Jos, Le Cours Blossac aux Sables-d'Olonne, Les Sables-d'Olonne, Imprimerie de l'Étoile de la Vendée, 1905, p. 18.

44. Arch. dép. de Loire-Atlantique, 534 S 90, Rapport de M. Bonnisseau, ingénieur en chef des Ponts-et-Chaussées, sur la construction d'un boulevard maritime à Pornichet, du 7 janvier 1926 . 
circule activement et notamment les propriétaires de villas la traversent pour aller à la plage ou pour accéder à la grande route [... ${ }^{45}$. " Il s'agit du boulevard de la station. En 1937, M. Marsat se préoccupe toutefois du piétinement des baigneurs escaladant en tous sens les talus de la dune ${ }^{46}$. La dune risque ainsi de perdre sa fonction de protection contre l'érosion littorale qui peut, à terme, nuire aux habitations. Mais, comme l'a déjà remarqué Gaëlle Delignon pour les stations de Saint-Malo et Paramé, la fonction défensive du remblai est facilement remplacée dans les esprits par la fonction balnéaire de la promenade ${ }^{47}$. En effet, la mer de la saison d'été est moins souvent terrible que celle de l'hiver, le bord de mer peut donc devenir la colonne vertébrale des villes littorales acquises à l'activité balnéaire.

Le littoral s'est transformé. Il est devenu le front de mer, où se dresse nettement une ligne entre terre et mer. D'une zone répulsive, il est devenu la zone attractive des communes balnéaires. D'un espace en réserve, il est devenu un lieu d'habitation, voire même le lieu idéal d'habitation. L'État et les sociétés littorales ont fait en sorte que cet espace particulier soit intégré à la terre, en fixant les dunes, en cadastrant les parcelles. Partant, c'est le lien avec la mer qui s'est renforcé. De nouveaux usages de la mer sont apparus. Elle est devenue un espace de repos servant à retrouver la santé, et un espace de jeu, profitable pour garder la santé. C'est en bord de mer que se font tous les profits. Voir la mer devient plus important qu'être sur mer. Les populations littorales sont donc logiquement exclues de cette vision, mais elles ne sont pas absentes. Elles ont su intégrer cet espace dans un nouvel ordre économique. Les terrains constructibles étant les terrains à plus haute valeur, il est normal qu'ils aient été vendus, quitte à délaisser les activités traditionnelles. Certains habitants se sont rapidement convertis, en construisant pour eux-mêmes des maisons disponibles à la location saisonnière. D'autres se sont orientés vers d'autres régions de la commune, profitant du pécule obtenu. Tous n'ont pas été nécessairement gagnants mais, favorisée par un afflux d'argent et de modernité, l'activité balnéaire ne pouvait que trouver un écho parmi les sociétés littorales. Il serait faux toutefois de généraliser. Toutes les communes littorales ne sont pas devenues des stations balnéaires. Le maire du Tour-du-Parc en 1939 déplore que « les ressources touristiques sont nulles. La côte seule, favorable à la pêche, mais dépourvue de plage méritant vraiment ce nom, peut attirer quelques amateurs ${ }^{48}$ ". Les réponses selon les communes ont été également différentes. Si certaines se sont orientées délibérément vers l'activité balnéaire, comme La Baule ou Saint-Jean-de-Monts, elles ont aussi choisi

45. Arch. dép. de Vendée, 7 M 215, Rapport de M. Marsat, inspecteur des Eaux et Forêts, sur l'aliénation de la dune littorale de Fromentine, sans date.

46. Arch. dép. de Vendée, 7 M 223, Rapport de M. Marsat, inspecteur des Eaux et Forêts, sur la dune littorale de Fromentine, du 13 décembre 1937.

47. Delignon, Gaëlle, op. cit., p. 106-107.

48. Arch. dép. du Morbihan, T 1538, Lettre du maire du Tour-du-Parc au préfet du Morbihan, du 26 mars 1939. 
leur chance de développement. Peu savent que La Baule était au XIX ${ }^{\mathrm{e}}$ siècle sous l'autorité de la rurale Escoublac, dont elle n'a jamais été distraite : la commune n'a pris le nom de La Baule-Escoublac qu'en 1962 (avec une étape intermédiaire en 1924 avec Escoublac-La Baule). D'autres stations balnéaires ont tâché de préserver certaines activités littorales, comme les activités de pêche, de port de plaisance ou de transport maritime. La spéculation demeure toutefois et l'attrait du littoral pérennise la mode des bains de mer. Elle touche maintenant tout autant le front de mer que les terres plus à l'intérieur : il n'est plus rare de devoir traverser, non plus un désert de sable, mais un désert (l'hiver) de lotissements, de par et d'autre de la route qui mène aux plages. Le débat est pourtant apparu très tôt : dès 1868, Jules Verne s'apitoyait sur la perte de l'originalité de Pornic, " originalité [qui] disparaît de jour en jour, grâce aux villas d'assez mauvais goût qui l'encombrent $[\ldots]^{49}$ ". La pointe de Gourmalon à l'époque n'était pas lotie, il s'agit des villas qui se trouvent sur la rive droite de l'embouchure de la HautePerche, que l'on peut apprécier aujourd'hui depuis la corniche de la Noëveillard. Être une station balnéaire relève donc d'un équilibre difficile à trouver : il faut construire pour le devenir tout en préservant pour le rester.

\section{RÉSUMÉ}

L'urbanisation du littoral est souvent considérée comme prédatrice, massive, propre à détruire le milieu qu'elle occupe. Or, la dynamique de cette urbanisation, la spéculation, est relativement récente sur ce territoire. Au $\mathrm{XVIII}{ }^{\mathrm{e}}$ siècle, le littoral reste un espace répulsif. La spéculation naît d'un regard nouveau sur cet espace qui se transforme à partir du $\mathrm{XIX}^{\mathrm{e}}$ siècle. Fixation des dunes de sable et mise en place du cadastre tâchent de rattacher le littoral aux terres mais l'intrusion balnéaire, dont les débuts remontent en BretagneSud et en Vendée au début du XIX ${ }^{\mathrm{e}}$ siècle, occupent la première cet espace. La spéculation manifeste la motivation des touristes, étrangers ignorants des conséquences de leurs comportements. Le bord de mer, autrefois redouté pour ses tempêtes, devient le lieu où il faut être, il se transforme en front de mer, incapable de s'adapter à la fluctuation du trait de côte.

\section{ABSTRACT}

Littoral urbanisation is often considered as predatory and massive. It is also thought that it tends to destroy the environment. Nevertheless, the dynamics of the urbanisation, the speculation, is relatively recent. In the $18^{\text {th }}$ Century, the littoral is still a repulsive territory. Speculation starts with a new relation for this space, which starts to be transformed at the beginning of the $19^{\text {th }}$ Century. The dunes are fixed and a cadastre is created. All these reforms try to connect the littoral to the land, but seaside intrusion, which began in the South of Brittany and in Vendée in the early $19^{\text {th }}$ Century, settle in first. Speculation shows the tourists'interests, as strangers who ignore the consequences of their behaviour. The seaside, which was in the past feared for the storms, becomes the place where you must be, it turns into a seafront, unable to adapt to the fluctuation of the coastal line.

49. VERNE, Jules, Géographie illustrée de la France et de ses colonies, Paris, Hetzel, 1868, p. 359. 